Revue d'histoire de l'Amérique française

ZWB REVUE D.HISTOIRE DE L'AMÉRIQUE FRANÇAISE

\title{
Les grands thèmes de l'histoire de la famille aux États-Unis
}

\section{Tamara K. Hareven}

Volume 39, numéro 2, automne 1985

Histoire de la famille

URI : https://id.erudit.org/iderudit/304349ar

DOI : https://doi.org/10.7202/304349ar

Aller au sommaire du numéro

Éditeur(s)

Institut d'histoire de l'Amérique française

ISSN

0035-2357 (imprimé)

1492-1383 (numérique)

Découvrir la revue

Citer cet article

Hareven, T. K. (1985). Les grands thèmes de l'histoire de la famille aux États-Unis. Revue d'histoire de l'Amérique française, 39(2), 185-209. https://doi.org/10.7202/304349ar d'utilisation que vous pouvez consulter en ligne.

https://apropos.erudit.org/fr/usagers/politique-dutilisation/ 


\title{
LES GRANDS THEMES DE L'HISTOIRE DE LA FAMILLE AUX ÉTATS-UNIS
}

\author{
TAMARA K. HAREVEN \\ Department of History \\ Clark University et \\ Center for Population Studies \\ Harvard University
}

Les origines de l'histoire de la famille remontent principalement à la «nouvelle» histoire sociale des années soixante. Les travaux historiques sur la famille partagent avec cette nouvelle histoire sociale un certain nombre de caractéristiques. D'abord, ils visent à reconstituer les biographies des gens ordinaires plutôt que celles des membres de l'élite ou des personnes célèbres. Ensuite, ils s'efforcent de mettre les comportements individuels et collectifs en rapport avec les structures sociales et les mouvements sociaux. Enfin, ils considèrent leurs sujets d'étude comme des acteurs et non comme des victimes des transformations historiques. Une partie importante de ces travaux analyse les comportements et les perceptions à partir du vécu et du point de vue des gens ordinaires. L'histoire de la famille a cependant connu ses développements majeurs au contact des théories et des méthodes de la démographie, de la psychologie, de la sociologie et de l'anthropologie. En contrepartie, la production scientifique issue du domaine de l'histoire de la famille a contribué à réviser les thèses généralement admises dans ces disciplines sur le comportement familial, le développement des individus et le changement social.

L'histoire de la famille a donné à la connaissance de la famille contemporaine une épaisseur historique et a fait ressortir la complexité des relations entre la famille et les autres institutions confrontées aux transformations historiques. Depuis quelques années les historiens ont étendu leurs enquêtes sur l'analyse de la structure des familles et des ménages vers des sujets plus variés comme le mariage, les comportements sexuels, l'éducation des enfants, les relations avec la famille étendue et les rapports inter-générationnels.

La recherche en histoire de la famille est passée d'une notion de la famille comme groupe statique de résidence, appréhendé à un seul moment de son existence, à une conceptualisation de la famille comme processus opérant durant toute la vie de ses membres; de l'étude des structures de la famille ou du ménage en elles-mêmes à l'étude des relations entre la famille nucléaire et le réseau de la parenté étendue; et 
de l'analyse de la famille comme unité domestique distincte à une analyse de ses rapports avec le monde du travail, avec le monde de l'éducation, avec les institutions d'assistance sociale et les institutions pénales, et avec des phénomènes sociaux, comme, par exemple, les migrations.

Plus récemment, l'exploration des processus de prise de décision au sein de la famille a conduit à l'étude des stratégies et des choix effectués par les membres de la famille en tant qu'individus et par la famille en tant que groupe ${ }^{1}$. Afin de saisir le rôle de la famille dans la société ainsi que sa dynamique interne, les chercheurs se sont éloignés d'une conception de la famille comme unité d'analyse en elle-même pour se pencher sur ses rapports avec les autres phénomènes sociaux et avec les institutions, en mettant particulièrement l'accent sur le rôle de la famille dans les processus d'industrialisation et d'urbanisation ${ }^{2}$. Enfin, le recours au concept de cycle de la vie familiale, qui a grandement pénétré la recherche sur la famille depuis quelque temps, y a introduit la notion de développement, car il fait ressortir le lien entre l'évolution des familles et celle des individus d'une part et le contexte historique dans lequel ils évoluent d'autre part par l'analyse des cohortes et des groupes d'âge ${ }^{3}$.

\section{La famille et la structure des ménages}

Les recherches sur l'histoire de la famille ont débusqué nombre d'idées reçues dans le milieu des sciences sociales sur les comportements familiaux dans le passé: par exemple, la thèse selon laquelle les comportements démographiques et familiaux «modernes» (comme par exemple les mariages précoces, la limitation des naissances ou la mobilité de la population), seraient apparus avec l'industrialisation et que

\footnotetext{
1 Voir G. Elder, «Approaches to Social Change and the Family», dans J. Demos et S. Boocock, dir., Turning Points: Historical and Sociological Essays on the Family (Chicago, University of Chicago Press, 1978); G. Elder, «History and the Family: The Discovery of Complexity», Journal of Marriage and the Family, 43 (1981): 489-519; J. Modell, «Patterns of Consumption, Acculturation, and Family Income Strategy in Late Nineteenth-Century America», dans T. Hareven et Maris Vinovskis, dir., Family and Population in Nineteenth-Century America (Princeton, N.J., Princeton University Press, 1978).

${ }_{2}$ M. Anderson, Family Structure in Nineteenth-Century Lancashire (Cambridge, Cambridge University Press, 1971); T. Hareven, «Family Time and Industrial Time: Family and Work in a Planned Corporation Town, 1900-1924», Journal of Urban History, 1 (1975): 365-389; T. Hareven, «Family Time and Historical Time», Daedalus, 106 (1977): 57-70; T. Hareven, «The Dynamics of Kin in an Industrial Community», dans Demos et Boocock, Turning Points; T. Hareven, Family Time and Industrial Time (New York, Cambridge University Press, 1982); N. Smelser, Social Change and the Industrial Revolution (Chicago, University of Chicago Press, 1959).

G. Elder, «Family History and The Life Course», dans T. Hareven, dir., Transitions: The Family and the Life Course in Historical Perspective (New York, Academic Press, 1978); Elder, «Approaches to Social Change»; T. Hareven, «Cycles, Courses, and Cohorts: Reflections on the Theoretical and Methodological Approaches to the Historical Study of Family Development», Journal of Social History, 12 (1978): 97-109; M. Vinovskis, «From Household Size to the Life Course: Some Observations on Recent Trends in Family History", American Behavioral Scientist, 21 (1977): 263-287.
} 
l'organisation domestique la plus courante dans les sociétés pré-industrielles était la famille étendue, et souvent la famille-souche. Cette thèse soutenait que l'industrialisation avait détruit la famille-souche et l'avait remplacée par la «famille nucléaire isolée», une forme d'organisation familiale perçue comme étant plus fonctionnelle dans le cadre de la société industrielle «moderne» que la famille étendue «traditionnelle». A cette thèse s'ajoutait souvent l'idée que la famille, ainsi que la communauté dont elle faisait partie, vivaient de façon harmonieuse et que c'est l'industrialisation qui a fait disparaître les formes traditionnelles d'organisation de la famille.

Ces idées reçues n'ont pu résister aux analyses produites par l'histoire de la famille au cours des années soixante. Le démographe P. Laslett et son équipe ont démontré la prédominance de la structure familiale nucléaire en Europe de l'ouest avant l'ère industrielle et sa survie depuis au moins trois siècles ${ }^{4}$. (Rappelons que Laslett emploie le terme de «famille nucléaire» pour désigner un groupe domestique simple, composé des parents et de leur progéniture ou d'un couple sans progéniture, ou encore d'un parent vivant avec un ou plusieurs de ses enfants. La famille nucléaire ne comprend donc pas de parents éloignés. Il faut bien distinguer ici entre «famille» et «ménage». En effet, le ménage «nucléaire» n'est pas assimilable à la famille nucléaire, celleci pouvant faire partie d'un groupe domestique comprenant des personnes non apparentées).

Les historiens qui ont souligné la permanence de la «famille» nucléaire dans le temps ont en fait tiré leurs conclusions de l'observation de ménages plutôt que de familles. En attirant l'attention sur la continuité des structures familiales, ils ont malheureusement laissé croire à la permanence de la famille nucléaire plutôt que du ménage nucléaire. La famille nucléaire ne vivait pas dans l'isolement; ses liens de parenté s'étendaient au-delà du ménage ${ }^{5}$. La confusion entre famille et ménage provient du fait que, dans la plupart des travaux sur l'histoire de la famille, l'unité d'observation était l'unité de résidence.

L'existence du ménage nucléaire observée sur au moins trois cents ans, en Europe de l'ouest, et sa prédominance au sein des communautés américaines de l'époque coloniale ${ }^{6}$ ont amené le rejet de l'hypothèse «la nostalgie occidentale de la grande famille», selon l'expression de

\footnotetext{
$4 \quad$ P. Laslett et R. Wall, dir., Household and Family in Past Time (Cambridge, Cambridge University Press, 1972).

5 Anderson, Family Structure; P. Greven, Four Generations: Population, Land, and Family in Colonial Andover, Massachusetts (Ithaca, N.Y., Cornell University Press, 1970); Hareven, «The Dynamics of Kin»; Hareven, Family Time; V. Yans-McLaughlin, Family and Community: Italian Immigrants in Buffalo, 1880-1930 (Ithaca, N.Y., Cornell University Press, 1977).

6 J. Demos, A Little Commonwealth: Family Life in Plymouth Colony (New York, Oxford University Press, 1970); W. Goode, World Revolution and Family Patterns (New York, Oxford University Press, 1963); Greven, Four Generations.
} 
William Goode - d'après laquelle la société pré-industrielle aurait été composée de ménages étendus comprenant trois générations. On n'a pas non plus retrouvé ces ménages étendus dans les communautés américaines du 19e siècle. Dans la plupart des communautés américaines de l'époque coloniale, le ménage nucléaire était le type le plus courant. Cependant, les membres des familles maintenaient des liens étroits avec leur parenté. Les parents âgés qui avaient un fils adulte, comme Greven l'a remarqué à Andover, ne vivaient pas avec leur fils mais habitaient tout près, souvent sur la même terre, formant ce qu'on pourrait appeler une structure familiale «étendue modifiée» ${ }^{7}$. Dans les villes américaines du $19 \mathrm{e}$ siècle, c'est toujours le ménage nucléaire qui constitue la forme la plus courante. On ne retrouve que de 9 à 12 pour cent de ménages étendus ou complexes; seulement de 1 à 3 pour cent des ménages sont composés de personnes seules. Les autres ménages sont tous de type nucléaire ${ }^{8}$.

Cette première phase de la recherche en histoire de la famille fit donc ressortir très clairement que l'industrialisation n'a pas conduit à l'apparition de la famille nucléaire isolée ou à la dissolution de la famille étendue. De fait, certains travaux ont démontré que l'industrialisation et l'urbanisation ont pu accroître la fréquence des ménages étendus ${ }^{9}$. Dans l'ensemble, cependant, ces travaux sur la composition des ménages tiraient leurs conclusions de l'analyse des ménages à un seul moment de leur existence et supposaient donc que cette structure était statique. On a critiqué ce postulat en montrant que la structure du ménage évoluait en fonction des étapes de la vie de la famille, de l'organisation de la production domestique et des modes de transmission de la propriété ${ }^{10}$.

La persistance du ménage nucléaire ne doit cependant pas nous amener à croire que les ménages de la période pré-industrielle ou ceux du $19 \mathrm{e}$ siècle étaient identiques aux ménages nucléaires d'aujourd'hui. Le ménage «nucléaire» d'autrefois était beaucoup plus complexe que celui d'aujourd'hui, car il comprenait souvent des personnes non apparentées vivant avec les membres du groupe familial. Ces personnes des domestiques, des apprentis ou des personnes plus âgées à la charge de la famille - participaient aux multiples fonctions remplies par l'unité

\footnotetext{
7 Demos, A Little Commonwealth; Greven, Four Generations; D. S. Smith, «Parental Power and Marriage Patterns: An Analysis of Historical Trends in Hingham, Massachusetts», Journal of Marriage and the Family, 35 (1973): 419-428.

8 S. Blumin, «Rip Van Winkle's Grandchildren: Family and Household in the Hudson Valley, 1800-1860», dans T. Hareven, dir., Family and Kin in Urban Communities (New York, Franklin Watts, 1977); Hareven, «Family Time and Historical Time»; R. Sennett, Families Against the City: Middle-Class Homes of Industrial Chicago, 1872-1890 (Cambridge, Mass., Harvard University Press, 1970).

9 Anderson, Family Structure; Hareven, Family Time and Industrial Time.

10 L. Berkner, «The Stem Family and the Developmental Cycle of the Peasant Household: An Eighteenth-Century Austrian Example», American Historical Review, 77 (1972): 398-418; J. Goody, «The Evolution of the Family», dans P. Laslett et R. Wall, dir., Household and Family in Past Time; E. Hammel, «The Zadruga as Process», ibid.
} 
domestique familiale avant l'industrialisation. La présence de ces personnes donnait à la composition des ménages une allure qu'on retrouve rarement de nos jours ${ }^{11}$.

Le ménage d'autrefois servait tout à la fois de lieu de travail, de mécanisme de formation professionnelle et d'institution d'assistance sociale; en conséquence, le ménage comprenait des personnes non apparentées qui entretenaient différents rapports économiques et sociaux avec les membres de la famille composant le noyau du ménage. A l'époque pré-industrielle, ces membres non apparentés étaient d'habitude des apprentis ou des domestiques; ils pouvaient aussi être, à l'occasion, des personnes dans le besoin placées sous les soins de la famille par les autorités locales. Les jeunes gens qui quittaient leur foyer pour aller vivre dans un autre ménage parcouraient de la sorte un itinéraire vers l'âge adulte fort répandu. Au $19 \mathrm{e}$ siècle, avec le déclin de l'apprentissage, et particulièrement de cette forme d'apprentissage qui consistait à placer les apprentis dans le foyer des maîtres, ce sont surtout des domestiques, des pensionnaires et des chambreurs qu'on retrouve comme personnes non apparentées dans les ménages nucléaires ${ }^{12}$.

Cette présence de pensionnaires et de chambreurs au sein des ménages américains et européens au 19e siècle constitue peut-être la caractéristique principale de la structure des ménages à cette période. La présence de personnes apparentées a constitué un phénomène moins important. Ainsi, au milieu du 19e siècle, aux États-Unis, la proportion des ménages qui abritaient des personnes apparentées à la famille nucléaire n'était que de 8 à 15 pour cent, alors que 20 à 30 pour cent des ménages comprenaient des pensionnaires ou des chambreurs. Les ménages des milieux urbains comprenaient donc plus souvent des étrangers que des parents. La présence de pensionnaires et de chambreurs y était aussi commune au $19 \mathrm{e}$ siècle et au début du $20 \mathrm{e}$ que celle des domestiques et des apprentis l'avait été dans les ménages de l'ère préindustrielle. Vivre en chambre ou en pension constituait une étape dans la vie de beaucoup d'individus; cette étape, qui influait sur le développement des familles comme sur celui des personnes, faisait en outre partie du processus de migration ${ }^{13}$.

Les hommes et les femmes qui avaient quitté le domicile de leurs parents et qui n'étaient pas encore mariés trouvaient par ce moyen un logement à prix abordable et une atmosphère familiale. Ce type de logement leur fournissait l'équivalent d'un support familial sans les

\footnotetext{
$11 \quad$ P. Laslett, The World We Have Lost (Londres, Methuen, 1965), traduit sous le titre Un monde que nous avons perdu (Paris, Flammarion, 1969).

12 Demos, A Little Commonwealth; J. Modell et T. Hareven, «Urbanization and the Malleable Household: An Examination of Boarding and Lodging in American Families», Journal of Marriage and the Family, 35 (1973): 467-479.

${ }_{13}$ Modell et Hareven, «Urbanization and the Malleable Household».
} 
obligations ni la surveillance imposées par la famille. Ces immigrants entraient, par l'intermédiaire du chef du ménage où ils résidaient, dans des réseaux de relations qui leur donnaient accès à des emplois et à un appui social. Ce n'est donc pas l'effet du hasard si les migrants se retrouvaient comme chambreurs chez des personnes de même origine ethnique et souvent de la même ville ou du même village ${ }^{14}$.

Pour les chefs de ménage, accueillir des pensionnaires ou des chambreurs était une façon d'accroître les revenus de la famille et de retrouver en quelque sorte dans ces jeunes personnes leurs propres enfants qui avaient quitté le domicile familial. Accueillir des pensionnaires ou des chambreurs servait done à maintenir «la péréquation sociale à l'intérieur de la famille» ${ }^{15}$. Au Massachusetts, à la fin du $19 \mathrm{e}$ siècle, les familles ouvrières tiraient une partie appréciable de leurs revenus de l'hébergement de pensionnaires ou de chambreurs, ce qui pouvait remplacer ou, selon la situation économique des familles, accroître les revenus provenant du travail des femmes ou des enfants. Ceci était particulièrement important pour les familles dont les enfants avaient quitté le domicile paternel. Les revenus qu'apportaient les pensionnaires au ménage permettaient aux épouses de ne pas travailler à l'extérieur du foyer et aux couples plus âgés de maintenir leur ménage après le départ des enfants ${ }^{16}$. Si la fonction économique et sociale des pensionnaires et des chambreurs au sein des familles d'accueil demande à être étudiée davantage, il est d'ores et déjà acquis que la présence de personnes non apparentées dans les ménages était plus fréquente et plus importante pour les ménages que la présence de parents. Il reste cependant à établir l'influence spécifique exercée sur la famille par les étrangers et par les parents.

\section{Les réseaux de parenté}

Les historiens ont été lents à délaisser le ménage comme unité d'analyse et à se pencher sur les réseaux de parenté qui débordent le ménage. L'impression que les familles américaines du $19 \mathrm{e}$ siècle vivant en milieu urbain avaient peu de relations avec leurs parents provient du fait qu'il n'est possible d'observer dans les ménages qu'une faible partie de la parenté. Jusqu'à tout récemment, les historiens ont eu peu de choses à dire au sujet de ces réseaux de parenté. Les difficultés inhérentes à l'établissement des liens de parenté hors du cadre du ménage y sont pour beaucoup, de même que la tendance à confondre famille et ménage.

\footnotetext{
14 Ibid.

15 I. Taeuber, «Change and Transition in Family Structures», dans The Family in Transition (Washington, D.C., Fogarty International Center Proceedings, 1969).

16 Hareven, Family Time and Industrial Time; Modell et Hareven, «Urbanization and the Malleable Household».
} 
Cette tendance qu'ont eue les historiens des structures familiales du 19e siècle à concevoir la famille, à la manière de Parsons, comme groupe nucléaire isolé, est d'autant plus ironique que les sociologues insistent maintenant sur l'importance des réseaux de parenté et d'entraide dans la société américaine contemporaine. C'est le cas notamment de M. Sussman, L. Burchinal et E. Litwak ${ }^{17}$. Les liens avec la parenté étendue étaient encore plus communs au 19e siècle et au début du 20e; la parenté formait la pierre angulaire de l'assistance et de l'entraide sociales en temps de crise avant que l'État-providence n'assume ces fonctions.

A la suite des travaux de sociologues portant sur la société contemporaine, à cause aussi de l'influence exercée par l'étude d'Anderson sur les réseaux de parenté au Lancashire au $19 \mathrm{e}$ siècle, les historiens ont commencé à faire ressortir le rôle de la parenté au sein des mouvements migratoires. Ils ont aussi montré l'importance du rôle des parents comme source d'appui et d'entraide lors de l'adaptation des migrants à leurs nouveaux milieux. En Angleterre et aux États-Unis, le modèle dominant d'organisation familiale fut celui du ménage nucléaire inséré dans un réseau de relations avec des parents résidant hors du ménage. La tendance générale - qu'on peut supposer être la stratégie préférée - était d'incorporer dans le ménage des personnes non apparentées et de conserver avec les parents vivant en dehors du ménage des liens d'assistance réciproque. C'est chez des parents vivant à proximité, plutôt qu'auprès de personnes non apparentées, qu'on cherchait des secours lorsque le besoin s'en faisait sentir ${ }^{18}$. Les ménages nucléaires font donc partie de réseaux de relations tissés par des liens de parenté. Dans un système où prévalait l'insécurité économique et en l'absence d'autres formes institutionnelles d'entraide, l'autonomie même de la famille reposait sur des relations de réciprocité avec des parents.

La parenté jouait de plus un rôle fondamental de médiation entre la famille et les autres institutions. Les parents organisaient les déplacements, facilitaient l'installation des nouveaux arrivants, les aidaient à trouver du travail et, comme il a été dit, contribuaient à amortir les secousses de l'adaptation aux nouveaux milieux. Les déplacements des individus et des familles vers les villes se firent par migration en chaîne, sous l'égide et avec l'appui des parents.

L'étude des milieux ouvriers de Manchester, au New Hampshire, illustre bien la persistance des liens de parenté lors du passage du milieu

\footnotetext{
17 E. Litwak, «Geographical Mobility and Extended Family Cohesion», American Sociological Review, 25 (1960): 385-394; M. Sussman et L. Burchinal, «Kin Family Network: Unheralded Structure in Current Conceptualization of Family Functioning», Marriage and Family Living, 24 (1962): 231-240.

18 Anderson, Family Structure; Hareven, «The Dynamics of Kin»; Hareven, Family Time and Industrial Time.
} 
rural au milieu industriel. A la fin du siècle dernier et au début du siècle présent, les réseaux de parenté ont servi d'instrument de médiation efficace avec le système industriel moderne ${ }^{19}$. Chez les immigrants canadiens-français, en particulier, les parents jouèrent un rôle crucial dans le recrutement de la main-d'oeuvre, l'organisation des mouvements migratoires vers le milieu industriel, l'appui aux nouveaux arrivants et l'affectation des travailleurs à l'intérieur de l'usine. Les parents constituaient le principal intermédiaire entre les travailleurs et leur employeur. Les réseaux de parenté facilitaient également les déplacements de travailleurs d'une ville industrielle à une autre. Dans les usines de textiles, les parents servaient à protéger les emplois, à initier les jeunes et les nouveaux venus aux méthodes de travail, et à trafiquer les machines ou les règlements pour ralentir le rythme de travail lorsque l'employeur forçait la cadence ${ }^{20}$.

Cette analyse des conditions de travail à l'intérieur d'une importante entreprise industrielle révèle jusqu'à quel point les liens entre les familles des travailleurs et l'entreprise étaient des liens de réciprocité. L'employeur, tout comme les travailleurs, utilisait la parenté comme intermédiaire principal. La famille formait ses membres au travail en usine et les protégeait contre les heurts et les bouleversements du milieu de travail. Ce faisant, la famille imposait ses traditions culturelles aux méthodes de travail, aux relations entre les secteurs de travail, aux relations entre travailleurs et aux relations entre les travailleurs et l'employeur.

En même temps que les parents formaient les jeunes travailleurs ou les immigrants fraîchement arrivés à la technologie et aux méthodes de travail industriel, à la discipline de travail et aux relations sociales entre travailleurs, ils les initiaient aussi aux comportements collectifs de la classe ouvrière. Le rôle central de la famille dans ce processus de formation des travailleurs nous porte à considérer que c'est la famille bien insérée dans un réseau de solidarités aux ressources variées, davantage que la famille nucléaire isolée, qui était la plus susceptible de s'adapter au travail industriel. D'autre part, en facilitant l'adaptation des jeunes travailleurs et des immigrants sans nuire à leur mobilité, les réseaux de parenté répondaient autant aux besoins des employeurs qu'à l'intérêt propre de leurs membres ${ }^{21}$.

Parmi les populations immigrantes, la parenté n'exerçait pas ses fonctions essentielles qu'à l'échelle de la communauté locale; elle agissait aussi à une échelle plus vaste. Les liens de parenté jouaient bien sûr plus efficacement lorsqu'il s'agissait de composer avec les institu-

19 Hareven, «The Dynamics of Kin»; Hareven, Family Time and Industrial Time.

20 Hareven, «Family Time and Industrial Time»; Hareven, «The Dynamics of Kin»; Hareven, Family Time and Industrial Time.

21 Hareven, Family Time and Industrial Time. 
tions locales ou de résoudre des crises passagères. Les réseaux locaux de parenté tiraient leur force de leur stabilité. Ils s'étendaient toutefois à plusieurs localités et ces liens étaient mis à contribution lorsque la communauté locale traversait des périodes difficiles ou durant les périodes de migration ${ }^{22}$.

A Manchester, au New Hampshire, comme dans le Lancashire du milieu du 19e siècle, comme dans l'est de Londres au 20e, les réseaux de parenté forment une partie intégrante de la ville industrielle ${ }^{23}$. Par contraste, l'espace de relations des Canadiens français s'étendait du Québec à Manchester et rejoignait d'autres villes industrielles de la Nouvelle-Angleterre. En plus de servir de système de sécurité, ces relations à distance permettaient de faire l'apprentissage de différentes occupations, d'envoyer les fils à la recherche d'emplois meilleurs, ou de trouver des conjoints aux filles. Lors des fermetures d'usine, les réseaux de parenté continuèrent à jouer, permettant à ceux qui étaient venus à l'usine de chercher des emplois temporaires ou permanents ailleurs dans la région ou de retourner au Canada. Ces réseaux de parenté, qui couvraient la Nouvelle-Angleterre, servaient à dépanner les travailleurs privés de travail ou de logement ${ }^{24}$.

Les réseaux de parenté étaient donc des phénomènes marquants, soit au niveau local dans le milieu de travail et dans le quartier, soit à l'échelle plus vaste des échanges inter-régionaux ou internationaux. Les formes élémentaires de coopération entre parents ne se modifiaient pas à cause de l'éloignement; elles s'enrichissaient plutôt de nouveaux modes d'entraide et visaient des objectifs plus diversifiés. Dans certains cas, les mouvements migratoires intensifiaient les relations de parenté en leur assignant de nouveaux rôles; ils resserraient ainsi les liens entre les communautés d'origine et les communautés d'accueil.

La conception organique de la famille - conception sous-jacente aux rapports entre les membres de la famille nucléaire et à l'intérieur du réseau de parenté tout au long du 19e siècle et au cours des deux premières décennies du $20 \mathrm{e}$ - exigeait la subordination presque totale des désirs individuels aux besoins du groupe familial. La carrière de chacun devait s'insérer dans l'économie générale de la famille. Les besoins du groupe familial déterminaient en grande partie la composition du ménage et fixaient le moment des grands étapes de la vie ${ }^{25}$. De

\footnotetext{
22 Hareven, «The Dynamics of Kin»; Hareven, Family Time and Industrial Time.

23 Anderson, Family Structure; E. Bott, Family and Social Network: Roles, Norms, and External Relationships in Ordinary Urban Families (Londres, Tavistock, 1957); M. Young et P. Willmott, Family and Kinship in East London (Londres, Routledge \& Kegan Paul, 1957).

24 Hareven, Family Time and Industrial Time.

25 H. Chudacoff et T. Hareven, «Family Transitions and Household Structure in the Later Years of Life», dans T. Hareven, dir., Transitions; Chudacoff et Hareven, «From the Empty Nest to Family Dissolution», Journal of Family History, 4 (1979): 59-63; Hareven, «The Dynamics of Kin»; J. Modell, F. Furstenberg et T. Hershberg, «Social Change and Transition to Adulthood in Historical Perspective», Journal of Family History, 1 (1976): 7-32; Modell et Hareven, «Urbanization and the Malleable Household».
} 
cette soumission aux objectifs familiaux dépendait aussi la participation des enfants, des adolescents et des femmes mariées au marché du travail. Survivance de la société rurale, la conception organique de la famille rendit possible l'adaptation au mode de vie ouvrier.

\section{Le cycle de la vie familiale et les itinéraires individuels ${ }^{26}$}

Si l'on exclut quelques travaux portant sur la période coloniale, la plupart des recherches faites au début de l'histoire de la famille ne portaient que sur des familles ou des ménages observés à un seul moment de leur existence. Quelques études portaient à l'occasion sur plus d'un moment dans la vie des familles ou des ménages, mais de façon générale, ces travaux, faits à partir de recensements de ménages, supposaient une stabilité dans la structure des familles et celle des ménages. Cette méthode était inapte à révéler les différentes formes d'organisation domestique que les membres d'une famille pouvaient connaître au cours de leur vie. De nouveaux concepts, comme celui du cycle de la vie familiale, et des méthodes d'analyse différentes, comme l'analyse des itinéraires individuels, ont permis de mieux saisir l'évolution des groupes domestiques à travers le temps.

Dans la critique méthodologique des travaux sur la famille qu'il a publiée en 1972, L. Berkner attira l'attention des historiens sur l'importance des transformations vécues par les ménages et les familles au cours de leur existence, particulièrement en ce qui a trait à la transmission des patrimoines ${ }^{27}$. Berkner prit comme exemple l'Autriche du $18 \mathrm{e}$ siècle, mais des travaux du même genre portant sur le 19e siècle ont eux aussi montré que les ménages et les familles changeaient de structure au long de leur existence; les personnes qui les composaient participaient de même à plusieurs formes d'organisation domestique depuis leur naissance jusqu'à leur décès.

Les groupes domestiques constituaient en quelque sorte des plaques tournantes; on y entrait et on en sortait sous l'influence de décisions personnelles ou de facteurs extérieurs à la famille ${ }^{28}$. Les personnes qui vécurent au sein de ménages nucléaires à un moment donné de leur vie ont souvent fait partie, à d'autres moments, de ménages étendus. La composition des ménages variait dans le temps en fonction de la disponibilité du logement, des migrations ou des changements dans leur situation économique ${ }^{29}$.

\footnotetext{
26 Traduction de «family cycle « et de «life course» (N. du T.).

27 Berkner, «The Stem Family».

28 Hareven, «The Family as Process: The Historical Study of the Family Cycle», Journal of Social History, 7 (1974): 322-329.

29 Chudacoff et Hareven, «Family Transitions»; Modell et Hareven, «Urbanization and the Malleable Household».
} 
Le concept de cycle de la vie familiale a donc amené les historiens à reconnaître aux structures des groupes domestiques une flexibilité qui n'était pas apparue dans les études transversales. L'analyse des comportements familiaux devint une analyse diachronique, axée sur les séquences d'évolution de ces comportements, même lorsque les données utilisées n'étaient prélevées qu'à un seul moment dans le temps.

L'application du concept de cycle de la vie familiale à l'étude du passé devait cependant en faire ressortir certaines limites. On ne peut retrouver de façon systématique, dans les populations passées, toutes les étapes du cycle de la vie familiale telles que définies par les sociologues des populations contemporaines, à cause des taux élévés de mortalité qu'on retrouve au $19 \mathrm{e}$ siècle. Dans son étude du cycle de la vie familiale chez des femmes américaines de race blanche entre 1870 et 1930 , Uhlenberg a noté que la séquence classique du mariage, de la formation de la famille, de l'éducation des enfants, du départ des enfants du foyer paternel et de la survie du couple jusqu'à ce que les conjoints atteignent l'âge de cinquante ans ne s'appliquait jamais à une majorité de la population avant le 20e siècle, même si cette séquence était la plus commune ${ }^{30}$.

Il devenait alors important, pour les populations passées, d'établir les moments de transition d'un rôle à un autre pour les individus au sein des familles, les périodes de changement dans les fonctions remplies par la famille et les périodes de transformation des structures familiales. Au lieu de calculer la période de temps médiane écoulée entre les différentes étapes du cycle de la vie familiale, les historiens se sont mis à mesurer la vitesse à laquelle chaque cohorte effectuait le passage d'une étape à l'autre ${ }^{31}$.

Si le concept de cycle de la vie familiale demeure utile pour l'étude des étapes du développment de la famille, l'analyse des itinéraires individuels, quant à elle, a fait avancer la recherche de plusieurs façons. En premier lieu, cette méthode permet de suivre plus fidèlement les parcours parfois complexes et sinueux des familles et des carrières dans des populations du $19 \mathrm{e}$ siècle ${ }^{32}$. En second lieu, elle permet d'analyser les transitions d'une étape à l'autre du cycle de la vie familiale et les rôles propres à chaque étape, là où l'on retrouve ces étapes. En troisième lieu, cette méthode a l'avantage de mettre en évidence les liens

\footnotetext{
30 P. Glick, «The Family Cycle», American Sociological Review, 12 (1947): 164-174; Glick, «The Life Cycle of the Family», Marriage and Family Living, 17 (1955): 3-9; Glick, "Updating the Life Cycle of the Family», Journal of Marriage and the Family, 39 (1977): 5-13; Glick et R. Parke, «New Approaches in Studying the Life Cycle of the Family», Demography, 2 (1965): 187-212; P. Uhlenberg, «Cohort Variations in Family Life-Cycle Experiences of U.S. Females», Journal of Marriage and the Family, 36 (1974): 284-292; Uhlenberg, "Changing Configurations of the Life Course», dans T. Hareven, dir., Transitions.

31 Modell, Furstenberg et Hershberg, «Social Change and Transition».

32 Elder, «Family History and the Life Course»; Hareven, «Cycles, Courses, and Cohorts»; Vinovskis, «From Household Size to the Life Course».
} 
chronologiques entre les transitions vécues par les individus et les transformations de la famille comme groupe. L'analyse des itinéraires individuels constitue donc une méthode plus efficace pour rendre compte des complexités de l'évolution historique des familles.

Le concept d'itinéraire individuel, émanant de la sociologie du vieillissement, de la notion de cohorte en démographie et des techniques d'histoire de vie appliquées en sociologie et en psychologie sociale, est un concept essentiellement dynamique ${ }^{33}$. Il apporte des éléments théoriques qui permettent de mieux comprendre les relations qui existent entre le développement individuel et celui des familles dans des contextes historiques changeants. Son influence sur l'histoire de la famille s'est faite sentir dans trois domaines principaux, soit l'étude des liens dans le temps entre les phases des itinéraires individuels et les transformations du groupe familial, celle des rapports entre ces deux phénomènes et le contexte historique, et celle de l'effet des premières étapes de la vie sur les étapes subséquentes ${ }^{34}$.

Les historiens qui ont adopté cette méthode l'ont appliquée à l'analyse des relations diachroniques liant le passage d'une étape à l'autre dans la vie des individus et celle des familles à une série de comportements individuels. Ainsi, le début et la fin de la fréquentation scolaire, l'intégration au marché du travail, la migration, le temps du départ ou du retour au domicile paternel, le mariage, la formation d'un nouveau ménage et l'accession à la retraite étaient réglés, comme on a pu le constater, davantage par des considérations de stratégies et d'évolution familiales que par des choix individuels. Si l'évolution du droit et des institutions a pu modifier l'âge auquel les gens s'intégraient au marché du travail ou le quittaient, en décrétant l'instruction obligatoire, en réglementant le travail des enfants et en fixant l'âge de la retraite, il n'en demeure pas moins que les réactions de la population à ces mesures furent façonnées par leur héritage culturel et familial. A l'intérieur des contraintes imposées par l'économie ou le cadre institutionnel, c'est la famille et les priorités qu'elle se donnait qui déterminaient le moment du passage d'une étape à l'autre dans la vie des individus qui la composaient; cela était particulièrement vrai aux époques où la famille était le principal lieu de formation d'activité économique et d'entraide.

La famille d'origine comme celle de procréation subissaient des transformations de structure à l'occasion du départ des jeunes, de leur

\footnotetext{
33 P. Baltes, «Life-Span Developmental Psychology: Some Converging Observations on History and Theory», dans P.B. Baltes et O.G. Brim, dir., Life-Span Development and Behavior (vol. 2, New York, Academic Press, 1979); Elder, «Family History and the Life Course»; M. Riley, M.E. Johnson et A. Foner, dir., Aging and Society: A Sociology of Age Stratification (3 vols., New York, Russell Sage, 1972); B. Neugarten et G.O. Hagerstad, «Age and the Life Course», dans R.H. Binstock et E. Shavas, dir., Handbook of Aging and the Social Sciences (New York, Van Nostrand, 1976); Riley, «Aging, Social Change and the Power of Ideas», Daedalus, 107 (1978): 39-52.

34 Hareven, «Cycles, Courses, and Cohorts».
} 
mariage ou de la fondation d'un nouveau ménage ${ }^{35}$. Le départ du domicile paternel était autant une décision familiale qu'une décision individuelle. De la même façon, le mariage, considéré aujourd'hui essentiellement comme le résultat de deux décisions individuelles, touchait trois unités familiales: la famille d'origine de chacun des conjoints et la nouvelle famille formée par le mariage. Au 19e siècle, l'âge au mariage était établi par un ensemble de facteurs économiques et sociaux, par les pratiques culturelles et par les contraintes imposées par les institutions. Lorsqu'il y avait remariage, faisant suite à un divorce ou à un décès, la famille du nouveau conjoint s'intégrait au réseau de relations du groupe domestique sans que la famille du conjoint précédent n'en sorte nécessairement.

Les dates des transitions majeures de la vie ont changé depuis le $19 \mathrm{e}$ siècle. Ces changements sont le résultat de transformations démographiques et de l'évolution des institutions autant que de l'influence de l'héritage culturel des familles. Selon Uhlenberg ${ }^{36}$, l'évolution démographique des cent dernières années a eu pour effet d'homogénéiser les profils des itinéraires individuels des Américains et a grandement accru l'espérance de vie du groupe familial. La chute de la mortalité, particulièrement chez les jeunes, a permis à une plus grande partie de la population d'assumer les différents rôles définis à l'intérieur de la famille et - sauf en cas de divorce - de faire partie d'un groupe familial pour toute leur vie. Les enfants avaient donc de meilleures chances de parvenir à l'âge adulte sans perdre leurs parents ou leurs frères et soeurs ${ }^{37}$. De même, la probabilité qu'une femme survive avec son mari jusqu'à l'établissement des enfants s'est accrue constamment depuis la fin du $19 \mathrm{e}$ siècle et le début du $20 \mathrm{e}^{38}$. En même temps, comme les femmes se mariaient plus tôt et que leurs fonctions maternelles se terminaient elles aussi plus tôt au cours de leur vie, l'accroissement de la longévité féminine s'accompagnait d'un allongement de la période suivant le départ des enfants. Comme l'espérance de vie des femmes était plus élevée que celle des hommes, les femmes survivaient à leur mari plus longtemps qu'auparavant. Inversement, une espérance de vie plus faible et une tendance plus forte au remariage chez les veufs signifiait que les hommes faisaient presque toujours partie d'un couple lorsque survenait leur décès ${ }^{39}$.

Le phénomène le plus marquant dans l'évolution des itinéraires individuels a été l'apparition d'une phase «post-parentale», où le couple

\footnotetext{
35 L. Glasco, «The Life Cycles and Household Structure of American Ethnic Groups: Irish, Germans, and Native-Born Whites in Buffalo, New York, 1885», dans Hareven, dir., Family and Kin; Hareven, «The Family as Process».

36 Uhlenberg, «Changing Configurations».

Uhlenberg, «Cohort Variations».

Ibid.

39 Glick, «Updating the Life Cycle»; Glick et Parke, «New Approaches».
} 
vit seul, les enfants ayant quitté le ménage. Ce qu'on a appelé en anglais le empty nest ${ }^{40}$ est devenu plus commun dans la phase adulte et dans la phase de vieillissement du couple avec la chute de l'âge au mariage et du nombre d'enfants, leur arrivée précoce dans la vie du couple et leur départ accéléré ${ }^{41}$.

$\mathrm{Au} 19 \mathrm{e}$ siècle, avant que ne se produisent les chutes de mortalité et de fécondité, cette phase était plus courte ou n'était jamais atteinte, car les familles étaient plus nombreuses, de sorte que le groupe domestique comprenait presque toujours un enfant, même rendu à l'âge adulte, ce qui contribuait à réduire le poids des difficultés financières ${ }^{42}$. Dans de telles familles, les plus jeunes apprenaient de leurs aînés ou des membres de leur parenté les rôles qu'ils avaient à jouer dans la famille, et inversement les plus vieux s'initiaient à leur rôle d'adulte en s'occupant de leurs cadets ${ }^{43}$.

Le passage d'une phase à l'autre du cycle de la vie familiale était alors moins tranché: la famille comprenait des enfants dont le plus jeune pouvait débuter l'école et le plus vieux se préparer au mariage. Les parents conservaient donc leur rôle de parent plus longtemps, souvent jusqu'à leur mort. Les pères vivaient rarement assez longtemps pour assister au mariage de leur troisième enfant. Moins formelle qu'aujourd'hui, la démarcation entre les différentes phases du cycle de la vie familiale s'accompagnait d'une plus grande fluidité dans leur chronologie. Ainsi le mariage n'impliquait pas toujours le départ du foyer paternel; de même, l'établissement des enfants ne réduisait pas nécessairement le groupe domestique au seul couple parental. Un enfant marié pouvait revenir au domicile de ses parents; les parents pouvaient aussi prendre des pensionnaires ou des chambreurs. On assiste, depuis les années 1970, à une résurgence de ces modèles de comportements familiaux: mariage retardé et grossesses tardives chez les femmes de carrière, le retour des jeunes adultes à la maison paternelle ou le prolongement de la période de co-habitation avec les parents.

Depuis le début du siècle, la convergence des rythmes de transition entre les périodes de la vie constitue l'évolution historique la plus marquante. Ceci est surtout évident dans le cas des étapes du passage à l'âge adulte: le départ du foyer paternel, le mariage et la formation d'un nouveau groupe domestique. J. Modell et ses collègues ont montré que ces périodes de transition sont devenues plus clairement définies, plus courtes, et plus rapprochées les unes des autres ${ }^{44}$. Ces transformations,

\footnotetext{
40 Littéralement, le «nid vide» (N. du T.).

41 Glick, «Updating the Life Cycle».

42 Chudacoff et Hareven, «From the Empty Nest to Family Dissolution»; Hareven, Family Time and Industrial Time; D. S. Smith, «Life Course Norms, and the Family System of Older Americans in 1900», Journal of Family History, 4 (1979): 285-298.

43 Hareven, «Family Time and Historical Time».

44 Modell, Furstenberg et Hershberg, «Social Change and Transition to Adulthood».
} 
qui se sont accompagnées de l'apparition de règles sociales et institutionnelles de passage, ont amené une ségrégation sociale par groupe d'âge et ont fait apparaître de nouvelles contraintes, tant à l'intérieur qu'à l'extérieur de la famille, dans l'établissement des temps de passage. Ceux-ci dépendent maintenant davantage des normes dictant les comportements de chaque groupe d'âge que de la situation particulière de chaque famille ${ }^{45}$.

Les grands changements esquissés ici ne tiennent pas compte des écarts chronologiques entre cohortes et des déphasages au sein de chacune. Ce domaine reste à explorer plus avant. N. Ryder a émis l'hypothèse que le changement social se produit lorsqu'une cohorte peut sentir des différences marquées entre ce qu'elle vit et ce qu'ont vécu les cohortes qui l'ont précédée ${ }^{46}$. Ces ruptures peuvent aussi se produire entre les différentes phases du cycle de vie d'une même cohorte. De telles expériences, combinées à l'effet différentiel de l'appartenance de classe, de l'appartenance ethnique et de l'appartenance locale, peuvent produire des variations majeures au sein d'une même cohorte.

Les historiens ont rarement eu l'occasion, jusqu'ici, de se pencher sur les effets cumulatifs des transitions d'une phase à l'autre des itinéraires individuels. Ils n'ont pu disposer de données sérielles comme celles qu'a colligées G. Elder sur les cohortes de Berkeley et d'Oakland, en Californie, dont les débuts coincident avec la période de la Crise $^{47}$. On peut cependant supposer que les itinéraires individuels sont fonction à la fois des circonstances historiques et de l'effet cumulatif des expériences vécues précédemment.

\section{Les stratégies familiales}

La production historique et sociologique sur la famille, qui a surtout examiné l'influence des institutions externes sur l'institution familiale, s'est ouvert des horizons nouveaux en prenant la lunette par l'autre bout. L'étude des stratégies familiales face aux contraintes du milieu et l'étude de la répartition des ressources au sein de la famille ont fait découvrir les directions empruntées par les familles pour prendre leur destinée en charge et jouer leur rôle d'agent de changement social. La recherche porte maintenant sur la dynamique interne des stratégies familiales: comment les familles planifiaient-elles leur développement, comment s'adaptaient-elles aux différentes phases de leur vie, comment réagissaient-elles au changement social? Comment les individus assumaient-ils les multiples rôles et obligations qui leur incombaient en

\footnotetext{
45 Ibid.

46 N. Ryder, «The Cohort as a Concept in the Study of Social Change», American Sociological Review, 30 (1965): 843-861.

47 G. Elder, Children of the Great Depression: Social Change and Life Experience (Chicago, University of Chicago Press, 1974).
} 
tant qu'enfants, parents, membres de réseaux de parenté, travailleurs? Comment harmonisait-on carrière personnelle et destinée familiale? Comment prenait-on les décisions à l'intérieur de la famille?

Ces questions commencent à faire l'objet de recherches, surtout en ce qui touche les aspects économiques de la vie familiale, comme les rapports avec le milieu industriel, l'utilisation de la main-d'oeuvre familiale et la répartition des ressources économiques à l'intérieur de la famille. Certains économistes ont représenté de façon exagérée la rationalité économique interne de la famille dans l'établissement des stratégies et dans le calcul de la valeur des prestations que les membres de la famille se fournissent mutuellement ${ }^{48}$. D'autre part, des historiens considèrent que les relations qu'entretient la famille avec la sphère économique sont fondées sur des activités délibérées de planification et de choix parmi les possibilités économiques qui s'offrent à elle. Cela ne veut toutefois pas dire que ces activités sont le fruit d'un calcul explicite. Dans ce domaine, le déclin du contrôle parental, celui du père en particulier, sur les phases de la vie des jeunes adultes a laissé à ceux-ci une indépendance plus grande; parallèlement, à l'intérieur du couple, l'ascendant du mari s'est effacé en faveur d'une relation plus équilibrée entre conjoints ${ }^{49}$.

Les recherches récentes se sont surtout employées à faire ressortir le poids des pratiques culturelles par rapport aux considérations d'ordre économique dans la prise de décision à l'intérieur de la famille. La prise de décision était considérée comme une entreprise collective qui portait sur une vaste gamme d'activités, depuis la transmission du patrimoine, la migration et la composition du groupe domestique jusqu'à la limitation des naissances et l'éducation des enfants. Cela impliquait une subordination des intérêts individuels à ceux de la famille comme groupe. Cette subordination se manifestait lors de la prise de décisions visant à maintenir la solvabilité de la famille, à faire l'achat d'une maison, à faciliter l'ascension professionnelle des enfants, à épargner pour l'avenir ou à assurer la retraite.

La participation des femmes et des enfants au marché du travail et la structure du revenu et des dépenses familiales ont fait l'objet d'études poussées. Le travail des épouses hors du foyer constituait un dilemme majeur pour les familles ouvrières. D'un côté, le travail des femmes à l'extérieur du foyer, comme celui des enfants, était nécessaire à la survie et à l'amélioration des conditions de vie de la famille. La contri-

G. Becker, A Treatise on the Family (Cambridge, Mass., Harvard University Press,

C. Degler, At Odds: Women and the Family in America From the Revolution to the Present (New York, Oxford University Press, 1980); Hareven, Family Time and Industrial Time D. B. Smith, Inside the Great House: Planter Family Life in Eighteenth-Century Chesapeake Society (Ithaca, N.Y., Cornell University Press, 1980); D. S. Smith, «Parental Power and Marriage Patterns». 
bution des femmes et des enfants à l'effort collectif de la famille faisait partie des normes culturelles que les travailleurs immigrants avaient acquises de leur milieu rural d'origine. De l'autre côté, le travail des mères de famille en dehors du foyer ne cadrait pas toujours avec ces normes culturelles et s'accordait mal avec les valeurs des classes moyennes de la société d'accueil. La famille devait donc composer à la fois avec les pressions économiques, le poids des valeurs traditionnelles et les valeurs de la société d'accueil.

Du 19e siècle jusqu'au début du 20e, la participation des femmes au marché du travail variait selon les étapes de la vie. Les femmes de la classe ouvrière entraient sur le marché du travail à leur adolescence et s'en retiraient après leur mariage ou après la naissance du premier enfant. Elles retournaient rarement sur le marché du travail après la naissance du dernier enfant, comme cela se produisait dans les années 1950 et 1960 . Les mères qui travaillaient à l'extérieur du foyer le faisaient de façon intermittente tout au long de leur période de fertilité. De fait, la plupart des femmes mariées, et surtout celles qui avaient des enfants, restaient hors du marché du travail, sauf dans les villes du textile, où l'emploi était majoritairement féminin ou familial ${ }^{50}$.

C'est d'abord vers le travail des enfants que se tournaient les familles dont le revenu était insuffisant ou le père malade, en chômage, ou parti, car le travail des enfants constituait la forme la plus stable de revenu additionnel pour la famille ${ }^{51}$. Les familles n'y avaient cependant pas toutes recours de la même manière. C. Goldin a montré comment, à la fin du $19 \mathrm{e}$ siècle à Philadelphie, les stratégies familiales produisirent dans ce domaine des différences marquées. Le travail des enfants variait selon le revenu de la famille, l'âge, le sexe, et la participation au marché du travail des autres enfants, ou l'absence d'un des parents. La mise au travail des plus jeunes enfants était fonction de la participation de leurs aînés au marché du travail. Les filles étaient moins portées à être envoyées au travail si elles avaient des frères aînés qui travaillaient; on peut voir dans ce phénomène une manifestation évidente de préférences dictées par des normes culturelles. Ces préférences se remarquent aussi à la réticence plus grande à faire travailler les femmes et les enfants dans les familles de souche américaine que dans les familles d'immigrants ${ }^{52}$.

\footnotetext{
50 K. Mason, M. Vinovskis et T. Hareven, «Women's Work and the Life Course in Essex County, Massachusetts, 1880», dans Hareven, Transitions.

C. Goldin, «Family Strategies and the Family Economy in the Late Nineteenth Century: The Role of Secondary Workers», dans T. Hershberg, dir., Philadelphia (New York, Oxford University Press, 1981); Hareven, «The Dynamics of Kin»; Hareven, Family Time and Industrial Time; Mason, Vinovskis et Hareven, «Women's Work and the Life Course»; M. Haines, «Industrial Work and the Family Cycle, 1889-1890», dans P. Uselding, dir., Research in Economic History (vol. 4, Greenwich, Conn., JAI, 1981)

Goldin, «Family Strategies».
} 
L'effet des préférences culturelles et des contraintes d'ordre économique se manifeste aussi dans les différences selon l'origine raciale. A Philadelphie, les femmes de race noire, qu'elles soient mariées ou veuves, travaillaient davantage à l'extérieur du foyer que les femmes de race blanche. On peut y voir l'effet de la pauvreté plus grande des familles de race noire; cependant, les enfants de race noire étaient moins souvent envoyés au travail que les enfants de race blanche, ce qui peut être le signe d'un désir de garder les enfants à l'école le plus longtemps possible au prix d'une tolérance plus grande du travail des femmes ${ }^{53}$.

Si l'on accepte de considérer ces comportements comme le résultat de stratégies familiales, on peut en inférer que les familles préféraient faire travailler les enfants, ou prendre des pensionnaires et des chambreurs, ou faire les deux à la fois, avant de recourir au travail des épouses, même si les familles les plus démunies n'avaient d'autre choix que d'adopter les trois méthodes. On peut aussi lire dans ces comportements la perception du travail des enfants comme élément essentiel du budget familial, alors que le travail des femmes représentait plutôt un revenu d'appoint. Cependant, malgré les réticences à faire travailler les femmes, leur participation au marché du travail était beaucoup plus élevée que ce que laissent supposer les données tirées des recensements. La participation des femmes au marché du travail fluctuait selon les grossesses, selon les besoins de la famille et selon les possibilités $\mathrm{d}^{\prime}$ emploi ${ }^{54}$. La tendance des femmes mariées à travailler dans le secteur des textiles ou des produits alimentaires reflétait à la fois une disponibilité plus grande d'emplois pour les femmes dans ces secteurs et une prédilection d'ordre culturel à faire travailler les femmes et les filles dans des secteurs se rapprochant des travaux domestiques assumés par les femmes ${ }^{55}$. De plus, lorsque cela était possible, les femmes et les filles avaient tendance à se trouver du travail dans des entreprises qui employaient d'autres membres de la famille. Le fait de travailler ensemble étendait le champ d'action de la famille au milieu du travail et permettait aux plus vieux de surveiller et de protéger les plus jeunes, les filles en particulier. Par ailleurs, même les femmes mariées qui poursuivaient une carrière de façon continue avaient tendance à considérer leur travail comme un revenu d'appoint pour la famille plutôt qu'un revenu de base ${ }^{56}$.

La majorité des travaux récents qui portent sur les stratégies familiales utilisent des données transversales, qui ne permettent pas de suivre l'évolution des stratégies familiales sur toute l'étendue des itinéraires

\footnotetext{
53 Ibid.

Hareven, Family Time and Industrial Time.

Yans-McLaughlin, Family and Community.

56 Hareven, Family Time and Industrial Time; J. Scott et L. Tilly, «Women's Work and Family in Nineteenth-Century Europe», Comparative Studies in Society and History, 17 (1975): 36-64.
} 
individuels; on ne peut que comparer à un même instant des cohortes d'âge différent. Les autres sources utilisées - recensements et budgets familiaux - ne permettent pas non plus d'appréhender directement les perceptions ou les ordres de priorité des membres des familles; tout au plus peut-on procéder par induction. Le processus de prise de décision à l'intérieur de la famille, tel qu'il était compris par ceux qui y participaient, échappe donc aux historiens. Seule l'enquête orale peut lever le voile sur ces questions.

\section{L'histoire de la famille et le changement social}

La production récente en histoire de la famille a profondément renouvelé nos connaissances des manières dont la famille a réagi aux transformations économiques et sociales; ces travaux ont aussi contribué, de façon plus générale, à éclairer les relations entre le changement social et les comportements individuels, étant donnée la position centrale de la famille comme intermédiaire entre les individus et la société. Dans cette perspective, on peut affirmer que la famille a servi à la fois d'instrument de changement social et de mécanisme d'adaptation à ces changements.

\section{Le rôle de la famille dans le processus d' industrialisation}

Ce double rôle de la famille apparaît le plus clairement lors de l'industrialisation. Jusqu'à récemment, la sociologie tenait pour acquis que la cellule familiale s'était effondrée sous l'effet de l'industrialisation. Selon les tenants de l'école de Chicago, l'avènement de la société industrielle et le passage de la campagne à la ville avaient coupé les liens qui rattachaient les individus aux réseaux traditionnels de parenté; les pressions engendrées par le travail en usine et la vie urbaine avaient amené la désintégration des groupes domestiques ${ }^{57}$. Même les sociologues qui n'adhéraient pas à cette théorie de la désintégration croyaient avec Parsons que la famille était passée d'une structure étendue à une structure nucléaire en réponse aux exigences de la société industrielle, la structure nucléaire étant la plus compatible avec ce type d'organisation sociale ${ }^{58}$. Un des principaux adeptes de cette thèse, W. Goode, conçoit la famille conjugale comme une structure au service de l'industrie ${ }^{59}$.

Ces thèses ont été complètement invalidées par les recherches historiques des dix dernières années. En fait, on a réussi à démontrer que

\footnotetext{
57 R. Linton, «The Natural History of the Family», dans R. Anshen, dir., The Family: Its Function and Destiny (Édition révisée, New York, Harper \& Row, 1959); W. Thomas et F. Znaniecki, The Polish Peasant in Europe and America (3 vols., Chicago, University of Chicago Press, 1918-1920); L. Wirth, «Urbanism as a Way of Life», American Journal of Sociology, 44 (1938): 1-24.

${ }_{58}$ T. Parsons et R. Bales, Family, Socialization, and Interaction Processes (Glencoe, Ill., Free Press, 1955).

59 Goode, World Revolution.
} 
l'industrialisation a pu renforcer les liens familiaux et augmenter la cohésion au sein de la famille. Le manque de logements, dans les régions industrielles, forçait les gens à habiter avec des parents, ce qui se traduisait par un nombre plus élevé de groupes domestiques étendus ${ }^{60}$. Loin d'obliger les jeunes à quitter le domicile des parents plus tôt, les possibilités d'emploi offertes par l'industrie leur donnaient l'occasion de rester à la maison, de travailler dans leur localité d'origine et de contribuer à l'entretien de la famille.

Dans son étude des débuts de la Révolution industrielle en Angleterre, Smelser a montré comment les usines de textiles embauchaient des familles entières comme unités de travail. Les pères de famille plaçaient leurs enfants, percevaient leurs salaires et faisaient régner la discipline à l'usine. En contrepartie, les usines dépendaient des groupes familiaux pour obtenir une quantité constante de main-d'oeuvre ${ }^{61}$. Vers 1830 , cependant, l'introduction de nouvelles machines amena une spécialisation accrue qui mit fin à ce type de travail familial. Le groupe domestique survécut cependant sous différentes formes comme groupe de travail tout au long du 19e siècle. Aux États-Unis, dans le secteur des textiles, par exemple, la famille continua à se comporter ainsi, et la parenté demeura un réseau de relations essentiel même lorsque le milieu industriel devint plus complexe à la fin du $19 \mathrm{e}$ siècle et au début du $20 \mathrm{e}^{62}$.

La famille, comme on l'a vu, a servi à la fois d'instrument de changement social et de mécanisme d'adaptation à ces changements. Dans quelles circonstances la famille était-elle capable de transformer son environnement socio-économique? Dans quelles circonstances perdait-elle ce pouvoir de transformation? Dans quelle mesure le changement social forçait-il les familles à revoir leur ordre de priorités? Comment cette révision affectait-elle la dynamique des relations familiales? Telles sont les questions que se pose aujourd'hui l'histoire de la famille.

L'exemple des moulins de Manchester nous fournit quelques indices. Dans cette ville, la famille tenait une place centrale dans le processus de recrutement de la main-d'oeuvre, de sorte que sa marge d'autonomie était plus grande durant les périodes de pénurie de maind'oeuvre que durant les périodes de surplus ${ }^{63}$. Le rôle de la famille ne s'effaça cependant pas avec la chute de son influence dans le milieu de travail lors du déclin de l'industrie textile; la famille mit au point de nouvelles façons d'affronter les difficultés engendrées par le chômage, les grèves et la mort éventuelle de l'industrie textile. Il faudrait toutefois analyser de façon plus systématique les circonstances qui ont permis

\footnotetext{
60 Anderson, Family Structure; Hareven, Family Time and Industrial Time.

61 Smelser, Social Change.

62 Hareven, Family Time and Industrial Time.

63 Hareven, Family Time and Industrial Time.
} 
aux familles d'influencer leur milieu socio-économique et les contextes où les familles n'ont eu d'autre choix que de se plier aux situations nouvelles.

\section{L'émergence de la famille «moderne»}

Les grands schémas linéaires d'explication des transformations dans le comportement des familles étant désormais rejetés, de nouveaux questionnements se font jour. Si l'industrialisation n'a pas provoqué la première chute substantielle de la fécondité, si elle n'a pas donné naissance aux ménages nucléaires, si elle n'a pas entraîné de réorganisation pénible de la structure familiale, peut-on lui attribuer quelque effet que ce soit sur le comportement des familles? Si le ménage nucléaire a constitué la forme principale d'organisation des groupes domestiques en Europe de l'ouest depuis des siècles, jusqu'où faut-il remonter pour en retrouver les origines? Et, si l'on peut repérer certaines caractéristiques de la famille «moderne» avant la Révolution industrielle, quelles sont les causes des changements dans la structure et le comportement des familles depuis trois siècles?

Si les historiens ne croient plus que l'industrialisation ait produit un type nouveau de structure familiale, ils sont toutefois d'accord pour lui reconnaître un effet sur les fonctions remplies par la famille, sur les valeurs dont elle est chargée et sur la chronologie des passages d'une étape à l'autre de la vie. Quoique ces transformations n'aient souvent pas eu de liens directs avec l'industrialisation, elles découlaient de la restructuration de l'économie et du progrès de l'urbanisation qui accompagne l'arrivée de l'industrie. On est de plus d'accord pour affirmer que le principal changement amené par l'industrialisation a été le transfert des fonctions remplies par la famille vers d'autres institutions sociales. On peut utiliser la terminologie de Parsons et parler de processus de différenciation: «Lorsqu'une organisation sociale devient archaïque sous l'effet des changements historiques, elle se scinde... en deux rôles ou en deux organisations, ou en un nombre plus grand, qui opèrent de manière plus efficace dans le nouveau contexte historique.» ${ }^{64} \mathrm{La}$ famille pré-industrielle servait d'atelier, d'église, d'école, de maison de correction ou d'asile ${ }^{65}$. Au cours du dernier siècle et demi, ces fonctions ont été dévolues en grande partie à d'autres institutions. Lieu de production, le ménage est devenu lieu de consommation et d'éducation des enfants. La famille s'est retirée du milieu de travail, trouvant refuge dans une intimité glorifiée; le monde du travail a rompu ses liens avec la famille et s'est bureaucratisé.

La famille s'est refermée sur elle-même, sur son intimité, dont elle a fait un idéal autant qu'une pratique. L'intimité du foyer et le clivage

64 Smelser, Social Change.

65 Demos, A Little Commonwealth. 
entre l'univers familial et le monde du travail sont devenus des caractéristiques fort prisées de la vie de famille ${ }^{66}$. Cet attachement à la vie domestique de la famille est en soi le résultat d'une longue évolution historique, qui débute avec la période moderne en Europe, et que P. Ariès a dépeint dans ces termes: «...la famille moderne se retranche du monde, et oppose à la société le groupe solitaire des parents et des enfants. Toute l'énergie du groupe est dépensée pour la promotion des enfants, chacun en particulier, sans aucune ambition collective: les enfants plutôt que la famille..${ }^{67} \mathrm{La}$ famille du $17 \mathrm{e}$ siècle, écrit l'auteur, se distingue de la famille moderne «...par l'énorme masse de sociabilité qu'elle conserve. Elle est, là où elle existe, c'est-à-dire dans de grandes maisons, un centre de relations sociales, la capitale d'une petite société complexe et hiérarchisée que commande le chef de famille» ${ }^{68}$.

Sous l'effet de la croissance économique et de l'industrialisation, la famille a perdu plusieurs de ses fonctions et s'est consacrée essentiellement à la consommation, à la procréation et à l'éducation des enfants. Comment la perte de ces fonctions et la diminution de la taille du ménage ont-elles modifié la dynamique interne des familles et la qualité des liens qui unissaient ses membres? Selon P. Ariès, la réduction des fonctions de la famille, son intimité grandissante, le déclin de la sociabilité et de l'intégration de la famille à la communauté constituent les phases caractéristiques de l'émergence de la famille moderne. Cette famille aura une structure nucléaire et entretiendra des rapports affectifs intenses; elle sera fière de son intimité et dévouée aux enfants. Ariès en conclut que ces transformations ont réduit les capacités d'adaptation de la famille et ont enlevé aux enfants l'occasion de grandir dans un milieu souple et d'observer un nombre varié de modèles de comportement. Cette interprétation demande à être soumise à des investigations plus poussées.

Comme la famille évolue plus lentement que les autres institutions sociales et qu'elle est autant un instrument de changement social que d'adaptation au changement, les historiens arrivent mal à fixer dans le temps les étapes de la transformation de la famille. On situe l'apparition de la «famille moderne», en Occident, entre 1680 et 1850 . P. Ariès et L. Stone ont privilégié la fin du $17 \mathrm{e}$ et le commencement du $18 \mathrm{e}$ comme moment critique, alors que E. Shorter retient plutôt la fin du $18 \mathrm{e}$ et le début du $19 \mathrm{e}$. Stone fait remonter la naissance de la «famille nucléaire

\footnotetext{
66 N. Cott, The Bonds of Womanhood: Woman's Sphere in New England, 1780-1835 (New Haven, Conn., Yale University Press, 1977); Degler, At Odds; B. Welter, «The Cult of True Womanhood, 1820-1860», American Quarterly, 18 (1966): 151-174.

67 P. Ariès, L'enfant et la vie familiale sous l'Ancien Régime (Paris, Seuil, 1973; édition

originale, Paris, Plon, 1960), 306.
Ibid.
} 
domestique fermée» ${ }^{69}$ à la période 1640-1800; les Américains, quant à eux, la situent comme Shorter à la fin du $18 \mathrm{e}$ et au début du $19 \mathrm{e}^{70}$.

L. Stone, P. Ariès et E. Shorter ont pris comme critère de la naissance de la famille moderne l'apparition de l'individualisme dans les sentiments. Selon eux, les caractéristiques essentielles de la famille moderne sont la dimension réduite du groupe domestique, le souci de l'intimité et de la vie privée, l'attention portée aux enfants, et, comme fondement, le lien affectif entre les conjoints et entre les parents et les enfants. Ces auteurs ont tous remarqué que l'intimité et l'attention portée aux enfants commandaient un déclin du rôle de la parenté, des voisins et des amis, ainsi qu'un isolement croissant de la famille par rapport à son milieu immédiat. De nos jours, les unions matrimoniales sont faites de liens affectifs entre les conjoints et ces liens naissent de l'attrait entre les personnes et de leurs pulsions sexuelles; elles ne dépendent plus d'alliances conclues par les parents. L. Stone et, à un moindre degré, $\mathrm{C}$. Degler considèrent que ce genre de famille ne peut entretenir des liens très forts avec la parenté.

Si les historiens acceptent généralement cette description de la famille moderne, on s'entend moins bien sur l'origine du phénomène. $\mathrm{P}$. Ariès, L. Stone et, de manière plus implicite, C. Degler l'attribuent à la petite noblesse et à la bourgeoisie; la famille moderne se serait ensuite «propagée» aux classes inférieures. Pour Shorter, au contraire, c'est du côté des paysans et des travailleurs qu'on doit regarder. Aux États-Unis, Degler perçoit l'apparition du phénomène au sein de la classe moyenne, d'où elle s'étendrait à l'ensemble de la société.

Les travaux sur ce sujet ont aussi pour trait commun une explication des transformations de la famille par l'idéologie et la culture plutôt que par des facteurs socio-économiques. Shorter est le seul à faire allusion au «capitalisme de marché» comme cause principale de l'apparition du sentiment familial mais, comme l'ont souligné les critiques, il n'explicite pas le lien entre l'évolution de l'économie et la transformation des relations à l'intérieur de la famille ${ }^{71}$. Stone, quant à lui, attribue l'origine du phénomène à un ensemble de causes, mais, tout comme Degler, il privilégie les aspects culturels et idéologiques avant les aspects socio-économiques ${ }^{72}$.

L'analyse des différences de classe dans les comportements familiaux reste encore à faire. Y a-t-il véritablement «propagation»des

\footnotetext{
69 Traduction littérale de «closed domestic nuclear family» (N. du T.).

70 Ariès, L'enfant et la vie familiale; Degler, At Odds; E. Shorter, Naissance de la famille moderne: XVIIIe-XXe siècle (Paris, Seuil, 1977); L. Stone, The Family, Sex, and Marriage in England, 1500-1800 (New York, Harper \& Row, 1977).

71 L. Tilly et M. Cohen, «Does the Family Have a History?», Social Science History, 6 (1982): 131-179.

72 Degler, At Odds; L. Stone, «Family History in the 1980 's», Journal of Interdisciplinary History, 12 (1981): 51-87.
} 
modèles de comportement d'une classe à une autre et, si c'est le cas, par quels processus une classe choisit-elle les comportements qu'elle empruntera à une autre classe ${ }^{73}$ ? Les transformations qui ont été observées dans les classes supérieures ont attiré davantage l'attention que la persistance de formes plus anciennes de comportement dans les autres classes sociales. Dans les familles ouvrières et les familles d'immigrants, on remarque des caractéristiques pré-industrielles, quoique modifiées $^{74}$. Il n'est pas du tout certain que les caractéristiques de la «famille moderne» des classes moyennes se retrouvent dans les familles des autres classes. La recherche sur la famille américaine n'est pas encore assez avancée pour établir de façon définitive des différences de comportement par classe ou par groupe ethnique. On sait déjà, cependant, que le souci de l'intimité, l'attention portée aux enfants et la distance prise par rapport à la parenté que l'on remarque dans les classes moyennes ne se retrouvent pas nécessairement dans d'autres classes ou parmi les groupes ethniques non-WASP ${ }^{75}$. Dans ce domaine, les historiens ont noté des différences importantes entre les classes et les groupes ethniques du $19 \mathrm{e}$ siècle.

Les différences les plus sensibles apparaissent dans les attitudes face à l'économie collective de la famille. Alors que les classes moyennes visaient à séparer les domaines du monde du travail de ceux de la famille, les familles de travailleurs et d'immigrants se considéraient solidairement responsables de la situation économique de la famille et reléguaient les liens affectifs au second plan.

Il est clair que les classes inférieures et les groupes ethniques nonWASP ont avec le temps eu tendance à adoter les modèles de comportement des familles des classes moyennes. J. Modell a montré comment les habitudes de consommation et les goûts des familles d'immigrants ont rejoint ceux des familles de souche ancienne ${ }^{76}$. Mais cette homogénéisation des styles de vie ne s'est pas faite de façon linéaire ou uniforme ${ }^{77}$. Il reste à examiner plus en détail comment elle s'est produite.

Les discontinuités dans l'évolution historique de la famille ont amené les historiens à délaisser les interprétations linéaires un peu simplistes et à se pencher, souvent à l'échelle d'une seule localité et pour une courte période, sur les liens entre les comportements familiaux, les processus sociaux et les institutions. Leurs travaux ont déjà apporté des révisions majeures aux thèses admises précédemment; le défi est main-

\footnotetext{
${ }^{73}$ Ariès, L'enfance et la vie familiale; Degler, At Odds; Shorter, Naissance; Stone, The Family, Sex, and Marriage.

74 Hareven, Family Time and Industrial Time; Scott et Tilly, «Women's Work».

75 «White Anglo-Saxon Protestant» (N. du T.).

76 Modell, «Patterns of Consumption».

77 Modell et Hareven, «Urbanization and the Malleable Household».
} 
tenant de tirer de ces recherches une synthèse plus systématique qui couvrirait des périodes plus longues.

\section{Conclusion}

A l'heure actuelle, la recherche sur l'histoire de la famille et du changement social a produit trois conclusions majeures. D'abord, elle a permis de voir que la famille est une institution agissante dans ses rapports avec son environnement économique, social et culturel. En second lieu, elle a révélé que l'évolution de la famille ne suit pas les découpages chronologiques traditionnels. En troisième lieu, elle a démontré que l'évolution des comportements familiaux n'a pas suivi le modèle de cheminement linéaire prévu par les théories de la modernisation. La rencontre de la famille et de la société urbaine et industrielle moderne n'a pas conduit de façon automatique à des comportements familiaux dits «modernes». Même si la famille a subi des transformations marquées en s'adaptant au monde industriel et urbain, les comportements des membres de la famille se sont «modernisés» plus lentement que ceux des travailleurs d'usine. En fait, les travailleurs ont pu s'adapter aux transformations dans la nature du travail en se repliant sur l'institution familiale, dont l'évolution plus graduelle ne présentait pas les mêmes traumatismes.

Traduction de José E. Igartua ${ }^{78}$

\footnotetext{
78 Nous sommes reconnaissant à Gérard Bouchard pour sa lecture attentive de la traduction et des suggestions fort utiles qu'il nous a formulées (N. du T.).
} 